\title{
HIERARQUIZAÇÃO DAS AÇÕES DE PROMOÇÃO DE SAÚDE MUNICIPAL PELA APLICAÇÃO DO MÉTODO MULTICRITÉRIO TOPSIS.
}

\author{
Antonio Ronaldo Madeira de Carvalho \\ FURB \\ Rua Antônio da Veiga, 140 - Blumenau/SC \\ ronaldo.madeira00@hotmail.com \\ Nelson Hein \\ FURB \\ Rua Antônio da Veiga, 140 - Blumenau/SC \\ hein@furb.br
}

\begin{abstract}
RESUMO
Vários são os desafios relacionados à gestão da saúde no Brasil, imersa em um cenário de profundas dificuldades. Diante desse contexto, o objetivo deste estudo é identificar por meio da aplicação do método TOPSIS o ranking de classificação das ações assistenciais de promoção de saúde pública do município de Araucária, região metropolitana de Curitiba/PR. Metodologicamente a pesquisa caracteriza-se como descritiva em relação a seu objetivo, quanto aos procedimentos pode ser considerada como pesquisa aplicada a partir do levantamento de dados secundário e quantitativa em relação à abordagem do problema, sendo analisada por meio de técnica multicritério (TOPSIS). Como resultado, pôde-se evidenciar os rankings das ações assistenciais de promoção de saúde pública do município estudado, a ação melhor classificada foi a (ação 8) que trata da ação anual de promoção ao uso racional de medicamentos elaborada e implementada, sendo assim, esta ação teve o melhor desempenho na secretária de saúde do município de Araucária. Logo a segunda e a terceira melhor ação classificada foi a (ação 6) que trata das ações intersetoriais realizadas com as secretarias e a (ação 14) que trata da instituição da gestão municipal do núcleo interno de regulação (NIR) sob responsabilidade do departamento de controle, avaliação e auditoria. Por fim, compreende-se que o método TOPSIS pode ser utilizado como uma ferramenta estratégica pelos gestores públicos, na tomada de decisão do planejamento municipal do SUS.
\end{abstract}

Palavra-chave: Gestão e Saúde Pública, Indicadores; Controle; Método TOSIS.

\begin{abstract}
There are several challenges related to health management in Brazil, immersed in a scenario of deep difficulties. In view of this context, the objective of this study is to identify, through the application of the TOPSIS method, the ranking of the public health promotion assistance actions of the city of Araucária, metropolitan region of Curitiba, Paraná State. Methodologically, the research is characterized as descriptive in relation to its objective, regarding the procedures can be considered as applied research from the secondary and quantitative data collection in relation to the approach of the problem, being analyzed by means of multi-criteria technique (TOPSIS). As a result, it was possible to highlight the rankings of public health promotion assistance actions of the studied municipality, the best classified action was the action (action 8) that deals with the annual action of promotion to
\end{abstract}


the rational use of medicines elaborated and implemented, this action had the best performance in the secretary of health of the municipality of Araucária. Therefore, the second and third best classified action was the action (action 6) that deals with the intersectoral actions carried out with the secretariats and (action 14) that deals with the institution of municipal management of the internal regulatory nucleus (NIR) under the responsibility of the department of control, evaluation and audit. Finally, it is understood that the TOPSIS method can be used as a strategic tool by public managers, in the decisionmaking of the SUS municipal planning

Keywords: Management and Public Health. Indicators. Control. TOPSIS Method

\section{INTRODUÇÃO}

O desenvolvimento e as conquistas no setor da saúde pública brasileira ocorreram a partir das influências de todo o contexto político-social e da evolução econômica pelos quais o Brasil passou ao longo da sua história, marcado por regimes distintos até a criação do Sistema Único de Saúde (SUS) vigente. O SUS pode ser considerado um dos maiores patrimônios e conquistas sociais do povo brasileiro, desde a Constituição de 1988. Apresenta princípios que priorizam a democratização nas ações e serviços da saúde que passam a ser universalizados e descentralizados, seguindo uma mesma doutrina e os mesmos princípios organizativos, em todo o território nacional.

A compreensão do SUS implica em entender a saúde como um direito social de cidadania, que equivale ao direito à própria vida, no qual as ações e serviços devem ser assegurados pelo Estado e geridos sob a responsabilidade das três esferas autônomas de governo (federal, estadual e municipal). Esta estrutura nacional sistematizada é composta por unidades, serviços e ações que interagem, objetivando um fim comum, baseado nos seguintes princípios: Universalidade, Integralidade, Equidade, Participação Social, Regionalização, Hierarquização, Resolutividade, Descentralização e Complementaridade do Setor Privado. Dentre todos os desafios para operacionalizar e gerir este sistema complexo, um dos aspectos mais importantes é o que se refere ao financiamento das ações e serviços de saúde, em virtude da sua característica de governança compartilhada e interdependência tripartite (união, estados e municípios). Embora a legislação da saúde obrigue as três esferas de governo a realizarem planejamento sistemático, ascendente e participativo, indicando inclusive instrumentos próprios para a elaboração de tal planejamento, o que se observa é uma dificuldade em compatibilizar os instrumentos de gestão do SUS aos instrumentos de gestão de governo.

A gestão do SUS ainda é afetada pela recorrente descontinuidade de ações entre as trocas de governos, pela instabilidade do cenário político e pela baixa qualificação e profissionalização de seus gestores, na aplicação de ferramentas e métodos eficazes de avaliação, controle e tomada de decisão. Os principais instrumentos de planejamento e gestão municipal do SUS são o Plano Municipal de Saúde (PMS) e a Programação Anual de Saúde (PAS), que são elaborados quadrienalmente e anualmente, respectivamente, e expressam os compromissos do gestor para com a sociedade no âmbito da saúde. Nestes instrumentos estão contidas as metas, indicadores e recursos que devem orientar a gestão na melhoria das ações e na condição de saúde da população local.

Neste contexto, o problema de pesquisa a ser desvelado é: como estão classificados os indicadores assistenciais de promoção de saúde pública do município de Araucária tendo como técnica de análise o método TOPSIS? Partindo destas breves reflexões, aponta-se para o objetivo deste estudo que é identificar por meio da aplicação do método TOPSIS o ranking de classificação das ações assistenciais de promoção de saúde pública do município de 
Araucária, região metropolitana de Curitiba/PR.

O estudo traz ainda, algumas contribuições, como a social, cujo objetivo é mostrar à sociedade civil e a comunidade acadêmica a importância de se trabalhar com os indicadores e as ações assistenciais de promoção de saúde pública, tratando que ambos precisam estar alinhados para objetivos comuns. Ainda, contribui com a literatura ao analisar uma perspectiva diferenciada, por meio de um ranking de classificação das ações assistenciais de promoção de saúde pública, identificando quais que estão melhores ou piores tendo como base os indicadores selecionados. Finalmente, os resultados permitem que gestores públicos avaliem oportunidades de fortalecimento das ações assistenciais de promoção de saúde pública do município de Araucária.

Diante desta breve introdução, parte-se para a fundamentação teórica, segunda parte deste estudo, visto que o artigo está dividido em seis partes, introdução, breve fundamentação teórica, procedimentos metodológicos, apresentação e análise dos resultados e por fim as considerações finais e referências.

\section{FUNDAMENTAÇÃO TEÓRICA}

Este tópico tem como propósito apresentar uma discussão a partir das pesquisas bibliográficas, cujos conceitos e enfoques são apresentados por autores a respeito das temáticas pesquisadas. Inicialmente serão apresentados conceitos sobre gestão em saúde pública, logo, parte-se para as discussões ligadas a gestão pública e planejamento em saúde, e por fim abordagens sobre instrumentos de gestão municipais do SUS e o plano de saúde do município pesquisado.

\subsection{GESTÃO EM SAÚDE PÚBLICO}

Desde a criação do Sistema Único de Saúde (SUS) pela Constituição Federal de 1988 (CF/88), considerada uma das maiores conquistas sociais, quando a saúde passa a ser um direito de todos e dever do Estado, apesar de todos os desafios, ocorreram avanços e inúmeras transformações na gestão da saúde pública, tanto em razão das transições políticas, como pelo indispensável aprimoramento da visão gestora e assistencial estabelecida ao longo do desenvolvimento do SUS.

A Lei $n^{\circ} 8.080$ de 19 de setembro de 1990, definiu os papéis institucionais de cada esfera governamental no plano da gestão da saúde, a estrutura de financiamento e as regras de transferência de recursos entre os diferentes níveis de governo, por meio dos Fundos de Saúde (CARVALHO; BARBOSA, 2010). A Lei nº 8.142 de 28 de dezembro de 1990 dispôs sobre a participação da comunidade na gestão do SUS e sobre as transferências intergovernamentais de recursos financeiros na área da saúde, instituindo os Conselhos de Saúde e conferiu status público aos organismos de representação de governos estaduais e municipais (CARVALHO; BARBOSA, 2010).

No contexto das realidades para a gestão do SUS, desde a sua criação, houve a necessidade da reorganização dos conceitos, estruturas instrumentais e da construção de novos processos e ferramentas de apoio ao planejamento da política de saúde pública brasileira. Diante de diversos desafios e entraves, os diferentes entes federativos têm encontrado ao longo da gestão situações problemáticas e adversas, sendo uma delas sob o aspecto do financiamento público. 
Conforme Santos (2009) o financiamento do SUS ainda é objeto de disputa, refletindo-se na instabilidade e insuficiência dos recursos alocados. De um modo geral, o subfinanciamento crônico coloca obstáculos à ampliação da cobertura e do acesso dos brasileiros às políticas e também à qualidade desse serviço (TEMPORÃO, 2008). Além da instabilidade e insuficiência do financiamento público para o SUS, a cada ano que passa, persistem problemas de gestão (PAIM, TEIXEIRA; 2007).

Em relação à gestão do SUS, Paim e Teixeira (2007) apontam também a falta de profissionalização de gestores, a descontinuidade administrativa, o clientelismo político e a interferência político-partidária no funcionamento dos serviços como condições que comprometem a reputação do SUS perante os cidadãos e a expectativa dos servidores públicos enquanto trabalhadores e partícipes de um projeto civilizatório do escopo da Reforma Sanitária Brasileira (PAIM, TEIXEIRA; 2007).

A Constituição brasileira possibilitou a conformação de um modelo para o sistema de saúde atual. Entretanto, outras medidas devem transformar o cotidiano das organizações e do trabalho em saúde. Novas formas de financiamento, novos instrumentos de análise da realidade, novos modos de estruturação de equipes que permitam agir em saúde, devem ser pensadas para se alcançarem princípios como equidade, integralidade e participação da comunidade (CAMPOS, 2003).

\subsection{Gestão Pública e Planejamento em Saúde}

A Constituição Federal de 1988 estabeleceu também os instrumentos de planejamento da gestão pública: Plano Plurianual (PPA), Lei de Diretrizes Orçamentárias (LDO) e Lei Orçamentária Anual (LOA), aos quais deveriam se submeter todos os órgãos da administração pública. Tais instrumentos passaram a permitir o planejamento e a definição das despesas de custeio e de capital em cada esfera de governo, contemplando o conjunto de objetivos e metas para orientar a alocação dos recursos públicos.

No Sistema Único de Saúde, a operacionalização do planejamento é definida pelas normativas legais sobre o tema que determinam as funções de três instrumentos obrigatórios: Plano de Saúde (PS), Programação Anual de Saúde (PAS) e Relatório Anual de Gestão (RAG). Silva, Santos e Mendes (2012) afirmam que, o PS, a PAS e o RAG são instrumentos interdependentes e indissociáveis que favorecem a dinamicidade e a constância do completo processo de planejamento.

O PS deve abordar as intenções (objetivos) e os resultados (metas) a serem buscados no período de quatro anos; a PAS deve operacionalizar as intenções expressas no PS, apontando o conjunto de ações voltadas à promoção, proteção e recuperação da saúde, bem como à gestão do SUS; e o RAG deve registrar os resultados alcançados com a execução da PAS e orientar eventuais mudanças que se fizerem necessárias.

O processo de planejamento e gestão em saúde pública segue a mesma lógica do processo na gestão governamental e para que ocorra em consonância aos demais instrumentos de gestão pública é fundamental que seja realizada a compatibilização entre os instrumentos de gestão da saúde, PS, PAS e RAG e os instrumentos de planejamento e orçamento de governo PPA, LDO e LOA.

Silva, Santos e Mendes (2012, p. 17) afirmam que:

O planejamento - e instrumentos resultantes de seu processo, como planos e relatórios - é objeto de grande parte do arcabouço legal do Sistema Único de Saúde (SUS), quer indicando processos e métodos de formulação, quer como requisitos para fins de repasse de recursos e de controle e auditoria. Em relação ao planejamento e a instrumentos que lhe dão expressão concreta, destacam-se, inicialmente, as Leis No 8.080/1990 e No 8.142/1990 (Leis Orgânicas da Saúde). 
O planejamento no Sistema Único de Saúde é uma função de gestão que cumpre um requisito legal, se traduzindo em um mecanismo primordial para assegurar os princípios constitucionais do SUS. Nele estão expressas as responsabilidades dos gestores de cada esfera de governo em relação à saúde da população do seu território, integrando, organizando e sistematizando as ações e serviços ofertados.

O planejamento está intrinsecamente relacionado à gestão estratégica e governança do SUS que objetiva reorientar os programas e os projetos governamentais para ampliar a eficiência, a eficácia e a efetividade da ação das políticas de saúde. Desta forma, para que as ações de planejamento possam contribuir para o aperfeiçoamento da gestão do SUS, os gestores devem lançar mão de ferramentas que possibilitem monitorar e avaliar os resultados alcançados e as estratégias empregadas.

O monitoramento e a avaliação por sua vez precisam ser processos periódicos, orientados pelas diretrizes, objetivos, metas e indicadores assumidos no Plano de Saúde. A escolha metodologias que irão orientar a avaliação e o monitoramento dependem do tipo de apreciação que se pretende realizar, uma vez que toda análise consiste na emissão de um juízo de valor acerca das características e dos resultados dos programas e das políticas de saúde.

\subsection{INSTRUMENTOS DE GESTÃo MUNICIPAIS DO SUS}

Na esfera municipal, o planejamento da saúde municipal e a execução de ações e serviços de saúde envolvem a utilização de instrumentos e ferramentas de gestão definidos legalmente, cujo cumprimento dos prazos é exigido, justamente por conformar um ciclo de atividades interdependentes, para garantir a prestação de contas sistematizada em instrumentos setoriais, que se relacionam entre si e entre os instrumentos de planejamento do governo e por fim, para assegurar a transparência do processo. Podem ser adotadas várias metodologias, em sua elaboração, mas o planejamento municipal deve ser sempre um processo participativo, envolvendo trabalhadores e usuários, além de ascendente, ou seja, partindo das unidades de saúde e da leitura das necessidades de cada território (CONASEMS, 2009).

Os instrumentos de planejamento e gestão do SUS estão previstos na Constituição Federal de 1988, na Lei $\mathrm{n}^{\circ} 8.080$ de 19 de setembro de 1990, decreto $\mathrm{n}^{\circ} 7.508$ de 28 de junho de 2011, na Lei Complementar no 141 de 13 de janeiro de 2012, e Portaria $n^{\circ} 2.135$ de 25 de setembro de 2013.

\subsection{Plano de Saúde do Município Pesquisado}

Para este estudo foram utilizados os dados do Plano Municipal de Saúde (PMS) do Município pesquisado, vigente para os anos de 2018 a 2021. O documento está disponível para acesso público no sítio eletrônico do município.

O município pesquisado localiza-se no estado do Paraná e está integrado à Região Metropolitana da capital Curitiba, distante $27 \mathrm{~km}$ do centro desta. Conforme o Censo Demográfico de 2010, é um dos 18 municípios paranaenses com população superior a 100.000 habitantes.

No município pesquisado, as portas de entrada aos serviços de saúde municipais distribuem-se em 21 pontos de acesso ao SUS, entre Unidades Básicas de Saúde - UBSs, Unidades Básicas Saúde da Família - UBSFs e unidades avançadas de atendimento das 
UBSFs. O município conta ainda com equipamentos de atenção especializada em saúde, Centros de Especialidades Médicas e Odontológicas (CEMO), Centro de Atendimento Psicossocial (CAPS), Unidade de Pronto Atendimento (UPA), Serviço de Atendimento Móvel de Urgência (SAMU) e um Hospital Municipal (HMA). De acordo com o IBGE, o número estimado de pessoas dependentes do SUS no município em 2017 é de 93.323 habitantes, aproximadamente $66 \%$ da população local.

O PMS do município apresenta um mapa estratégico com missão, visão e valores, três eixos estruturantes de gestão, sendo estes, gestão de pessoas, gestão de recursos e gestão dos processos e da inovação, com foco nos resultados para população que culminem na redução dos índices gerais de mortalidade e na melhoria do acesso e resolutividade das necessidades do cidadão. São apresentadas oito diretrizes gerais distribuídas entre políticas de saúde e níveis de atenção à saúde, sendo as diretrizes desmembradas em 18 objetivos estratégicos de gestão. Cada objetivo estratégico contém ações, metas e indicadores próprios, totalizando 32 ações, 89 metas e 91 indicadores.

Segundo a Rede Interagencial de Informações para a Saúde (Ripsa),

Em termos gerais, os indicadores são medidas-síntese que contêm informação relevante sobre determinados atributos e dimensões do estado de saúde, bem como do desempenho do sistema de saúde. Vistos em conjunto, devem refletir a situação sanitária de uma população e servir para a vigilância das condições de saúde. A construção de um indicador é um processo cuja complexidade pode variar desde a simples contagem direta de casos de determinada doença, até o cálculo de proporções, razões, taxas ou índices mais sofisticados, como a esperança de vida ao nascer. (RIPSA, 2008, p.13)

Os indicadores seriam subsídios essenciais para o planejamento e formulação de políticas públicas sociais em todas as esferas de governo, pois permitem monitorar os condicionantes e determinantes sociais de vida e bem-estar da população, permitindo a análise do poder público, da sociedade civil e promovendo dados para a investigação acadêmica (JANNUZZI, 2012).

"No campo aplicado das políticas públicas, os indicadores sociais são medidas usadas para permitir a operacionalização de um conceito abstrato ou demanda de interesse programático na área social” (JANNUZZI, 2012, p. 20).

Ainda de acordo com Januzzi (2012), os indicadores podem se classificar, especialmente para análise e formulação de políticas sociais, de acordo com a sua natureza entre: de recurso (indicador-insumo), de realidade empírica (indicador-produto) ou de processo (indicador-processo).

\section{PROCEDIMENTOS METODÓLOGICOS}

A pesquisa caracteriza-se como descritiva em relação a seu objetivo, quanto aos procedimentos pode ser considerada como pesquisa aplicada a partir do levantamento de dados secundário e quantitativa em relação à abordagem do problema, sendo analisada por meio de técnica multicritério (TOPSIS). Os dados foram obtidos a partir de documentos (relatórios) que foram coletados através da secretaria de saúde do município de Araucária, município da região metropolitana de Curitiba, estado do Paraná.

Foram selecionados para este estudo ações e indicadores integrantes da diretriz 3 do plano estratégico da secretaria de saúde que trata do "Fortalecimento das Ações de Promoção à Saúde". Com o plano municipal de saúde e a programação anual de saúde (PAS) formamse os principais instrumentos de planejamento municipal do SUS, no qual orientam o gestor público no processo de tomada de decisão no que tange a melhoria das ações e a qualidade da saúde prestada a população local. Na tabela 01 é possível observar as dez ações 
(integrantes da diretriz 3 que trata do "Fortalecimento das Ações de Promoção à Saúde") que se relacionam com indicadores capazes de construir parâmetros de desempenho e importância, dentro do processo de tomada de decisão dos gestores da secretaria saúde.

Tabela 1: Indicadores e parâmetros de análise

\begin{tabular}{|c|c|c|c|c|c|}
\hline \multirow[b]{2}{*}{ AÇ.̃AO } & \multicolumn{4}{|c|}{ INDICADORES E PARÂMETROS DE ANÁLISE } & \multirow[b]{2}{*}{ Orçamento } \\
\hline & $\begin{array}{l}\text { Impacto inter- } \\
\text { departamentos }\end{array}$ & $\begin{array}{l}\text { Impacto inter- } \\
\text { secretarias }\end{array}$ & $\begin{array}{l}\text { Impacto Popu- } \\
\text { lacional }\end{array}$ & $\begin{array}{l}\text { Desempenho } \\
\text { atual em relação } \\
\text { à meta }\end{array}$ & \\
\hline $\begin{array}{l}1 \text { - Acompanhamento das condicionali- } \\
\text { dades de saúde do programa bolsa fa- } \\
\text { mília. } \\
2 \text { - Ações de prevenção e promoção } \\
\text { em saúde do trabalhador rural por } \\
\text { equipe de ESF realizadas. } \\
\text { 3- Projetos relacionados à atenção } \\
\text { básica. } \\
4 \text { - Projetos voltados a estratégia de } \\
\text { saúde da família. } \\
5 \text { - Integração UBS na proposta de pre- } \\
\text { venção e promoção da saúde da popu- } \\
\text { lação considerando as zoonoses de re- } \\
\text { levância no município. } \\
6 \text { - Ações intersetoriais realizadas com } \\
\text { as Secretarias. } \\
7 \text { - Palestras de prevenção e controle } \\
\text { do Aedes por dias de chuva. } \\
8 \text { - Ação anual de promoção ao uso ra- } \\
\text { cional de medicamentos elaborada e } \\
\text { implementada. } \\
9 \text { - Ação intersetorial para prevenção } \\
\text { da gravidez na adolescência } \\
10 \text { - Ação de incentivo ao aleitamento } \\
\text { materno } \\
11 \text { - Ações relacionadas ao atingimen- } \\
\text { to de ocupação hospitalar mínima } \\
12 \text { - Implantação e execução de audito- } \\
\text { ria operacional in loco no Hospital } \\
\text { Municipal } \\
13 \text { - Institucionalização de protocolo } \\
\text { de interface com a regulação de urgên- } \\
\text { cia e de leitos com ações locais coor- } \\
\text { denadas pelo departamento de contro- } \\
\text { le, avaliação e auditoria } \\
14 \text { - Instituição da gestão municipal do } \\
\text { núcleo interno de regulação (NIR) sob } \\
\text { responsabilidade do departamento de } \\
\text { controle, avaliação e auditoria } \\
15 \text { - Implantação de equipe do progra- } \\
\text { ma melhor em casa }\end{array}$ & $\begin{array}{c}\text { Parâmetro de } \\
\text { Análise: Quanto } \\
\text { maior a } \\
\text { quantidade de } \\
\text { departamentos } \\
\text { envolvidos, mais } \\
\text { complexo e } \\
\text { relevante é a ação. }\end{array}$ & $\begin{array}{l}\text { Parâmetro de } \\
\text { Análise: Quanto } \\
\text { maior a } \\
\text { quantidade de } \\
\text { secretarias } \\
\text { municipais } \\
\text { envolvidas, mais } \\
\text { complexo e } \\
\text { relevante é a } \\
\text { ação. }\end{array}$ & $\begin{array}{l}\text { Parâmetro de } \\
\text { Análise: Quanto } \\
\text { maior a } \\
\text { população } \\
\text { atingida mais } \\
\text { relevante é a } \\
\text { ação. }\end{array}$ & $\begin{array}{c}\text { Parâmetro de } \\
\text { Análise: Quanto } \\
\text { menor o } \\
\text { desempenho em } \\
\text { relação à meta, } \\
\text { maior será o } \\
\text { grau de } \\
\text { importância } \\
\text { dado às ações. }\end{array}$ & $\begin{array}{c}\text { Indica o } \\
\text { volume de } \\
\text { recursos } \\
\text { alocados } \\
\text { para a } \\
\text { realização } \\
\text { da ação. } \\
\text { Parâmetro } \\
\text { de Análise: } \\
\text { Quanto } \\
\text { maior o } \\
\text { orçamento, } \\
\text { maior a } \\
\text { complexida } \\
\text { de e } \\
\text { relevância } \\
\text { da ação. }\end{array}$ \\
\hline
\end{tabular}

Fonte: Adaptado da secretaria de saúde de Araucária (2019)

Quanto ao método de tratamento dos dados, os rankings foram elaborados de acordo com o modelo de análise multicritério Technique for Order Preference by Similarity to an Ideal Solution (TOPSIS). Este modelo indica a melhor alternativa dentre as alternativas analisadas, e tem como princípio selecionar a alternativa mais próxima da solução ideal positiva e a mais distante da solução ideal negativa (BULGURCU, 2012).

Os procedimentos para o método TOPSIS podem ser classificados em seis passos. Inicialmente deve ser elaborada a matriz de decisão, construída com as variáveis analisadas no estudo, dispostas de acordo com alternativas e critérios, a matriz de decisão A é combinada por alternativas e critérios é descrita de acordo com a Equação 1. 


$$
\begin{gathered}
C_{1} \\
A=\ldots \\
A_{1} \\
A_{m}
\end{gathered}\left(\begin{array}{ccc}
x_{11} & \ldots & x_{n} \\
\vdots & \ddots & \vdots \\
x_{m 1} & \cdots & x_{m n}
\end{array}\right)
$$

(Equação 1)

Onde $A_{1}, A_{2} \ldots$ Am são alternativas viáveis, $C 1, C 2, \ldots$, Cn são critérios,xij indica o desempenho da alternativa Ai segundo o critério $\mathrm{Cj}$.

Depois de elaborada a matriz dos dados, parte-se para o passo 2 que é a padronização dos mesmos, a partir da mensuração de pesos para cada grupo de índice. (falar como foi construído os pesos - CV)

O terceiro passo é a determinação da solução ideal positiva $(\mathrm{A}+)$ e da solução ideal negativa (A-) para cada variável analisada, de acordo com a Equação 2 e Equação 3.

$$
\begin{aligned}
& A^{+}=\left(p_{1}^{+}, p_{2}^{+}, \ldots, p_{m}^{+}\right) \text {onde } p_{j}^{+}=\left\{\operatorname{Max}_{i} p_{i j}, j \in J_{1} ; \operatorname{Min}_{i} p_{i j}, j \in J_{2}\right\} \\
& A^{-}=\left(p_{1}^{-}, p_{2}^{-}, \ldots, p_{m}^{-}\right) \text {onde } p_{j}^{-}=\left\{\operatorname{Min}_{i} p_{i j}, j \in J_{1} ; \operatorname{Max}_{i} p_{i j}, j \in J_{2}\right\}
\end{aligned}
$$

Onde: onde $\mathrm{J}_{1}$ e $\mathrm{J}_{2}$ representam respectivamente o critério benefício e custo.

O quarto passo consiste no cálculo das distâncias de cada variável em relação a solução ideal positiva e a solução ideal negativa, a partir do qual determina-se a distância da solução positiva $\left(\mathrm{D}^{+}\right)$e a distância da solução negativa $(\mathrm{D}+)$ de cada variável, de acordo com a Equação 4 e Equação 5.

$$
\begin{gathered}
d^{+}=\sqrt{\sum_{j=1}^{n} w_{j}\left(p_{j}^{+}-p_{i j}\right)^{2}} \operatorname{com} \mathrm{i}=1, \ldots, \mathrm{m} \\
d^{-}=\sqrt{\sum_{j=1}^{n} w_{j}\left(p_{j}^{-}-p_{i j}\right)^{2}} \text { com } \mathrm{i}=1, \ldots, \mathrm{m} .
\end{gathered}
$$

Onde $\mathrm{W}_{\mathrm{j}}$ é o peso do atributo ou critério

De posse destas medidas, parte-se para o quinto passo, que diz respeito ao cálculo do coeficiente de aproximação de cada variável, ou seja, o desempenho global ou a pontuação final de cada alternativa, o mesmo é calculado de acordo com a Equação 6.

$$
\xi_{i}=\frac{d_{i}^{-}}{d_{i}^{+}+d_{i}^{-}}
$$

O sexto passo é a construção do ranking em relação às variáveis, o qual é elaborado a partir da classificação em ordem decrescente dos coeficientes de aproximação, ou seja, é a classificação do maior para o menor dos indicadores calculados no quinto passo. Diante da exposição dos procedimentos metodológicos, parte-se para a apresentação e análise dos resultados. 


\section{APRESENTAÇÃO E ANÁLISE DOS RESULTADOS}

Neste capítulo apresenta-se a descrição dos resultados, desta forma, a tabela 02 tangibiliza os indicadores apontados na tabela 1 , na medida em que é mensurado o desempenho de cada ação em relação aos quatro indicadores apontados como mais relevantes.

Tabela 02 - Indicadores de desempenho

\begin{tabular}{c|c|c|c|c|c}
\hline Ação & $\begin{array}{c}\text { Impacto Inter } \\
\text { departamentos }\end{array}$ & $\begin{array}{c}\text { Impacto } \\
\text { Inter } \\
\text { secretarias }\end{array}$ & $\begin{array}{c}\text { Impacto } \\
\text { Populacional }\end{array}$ & $\begin{array}{c}\text { Desempenho } \\
\text { Atual em } \\
\text { Relação a } \\
\text { Meta }\end{array}$ & Orçamento \\
\hline 1 & 4 & 3 & 12.926 & 9,5 & $\mathrm{R} \$ 35.000,00$ \\
\hline 2 & 4 & 3 & 3.727 & 0 & $\mathrm{R} \$ 55.000,00$ \\
\hline 3 & 4 & 3 & 93.323 & 0 & $\mathrm{R} \$ 38.000,00$ \\
\hline 4 & 4 & 3 & 93.323 & 5,3 & $\mathrm{R} \$ 52.000,00$ \\
\hline 5 & 2 & 5 & 141.410 & 0 & $\mathrm{R} \$ 90.000,00$ \\
\hline 6 & 7 & 5 & 141.410 & 0 & $\mathrm{R} \$ 60.000,00$ \\
\hline 7 & 2 & 6 & 141.410 & 0 & $\mathrm{R} \$ 9.000,00$ \\
\hline 8 & 7 & 6 & 141.410 & 0 & $\mathrm{R} \$ 74.000,00$ \\
\hline 1 & 7 & 19 & 7.670 & 0 & $\mathrm{R} \$ 80.000,00$ \\
\hline 11 & 4 & 19 & 2.255 & 0 & $\mathrm{R} \$ 46.000,00$ \\
\hline 12 & 4 & 3 & 141.410 & 9,8 & $\mathrm{R} \$$ \\
\hline 13 & & & & & $22.800 .000,00$ \\
\hline 14 & 5 & 5 & 141.410 & 3,3 & $\mathrm{R} \$ 20.000,00$ \\
\hline 15 & 3 & 5 & 141.410 & 0 & $\mathrm{R} \$ 38.000,00$ \\
\hline PESO & $\mathbf{0 , 3 9 9 4}$ & $\mathbf{0 , 1 9 8 4}$ & $\mathbf{0 , 2 6 7 9}$ & $\mathbf{0 , 0 8 8 4}$ & $\mathbf{0 , 0 4 5 6}$ \\
\hline $\mathrm{FOn}$ & & & & 0 & $\mathrm{R} \$ 24.000,00$ \\
\hline
\end{tabular}

Fonte: Dados da pesquisa, 2019

É importante ressaltar a hierarquização proposta, tem como base os indicadores e parâmetros contidos na tabela 1 , que serviram de premissas para o ranqueamento das ações propostas, estas por sua vez já definidas pelo plano estratégico da secretaria de saúde do município estudado. Não foi objetivo deste trabalho a construção de proposta para novas ações, além das já previstas pela secretaria de saúde estudada.

Após a exposição dos dados, percebe-se que na última linha da tabela 2 apresenta-se os pesos, ou seja, trata-se da valorização obtida através do cálculo do coeficiente de variação. Neste caso os dois indicadores que possuem o maior peso, são 1 - Impacto Inter Departamentos $(0,3994)$ e 2 - Impacto Populacional $(0,2679)$. Em relação às ações com maior impacto inter-departamentos, é possível observar que aparecem empatadas com 7 departamentos envolvidos as ações intersetoriais realizadas com as secretarias, a ação anual de promoção ao uso racional de medicamentos elaborada e implementada e a ação intersetorial para prevenção da gravidez na adolescência.

Em relação às ações como maior impacto no que diz respeito ao número de secretarias municipais envolvidas a ação inter-setorial para prevenção da gravidez na adolescência e a ação de incentivo ao aleitamento materno aparecem empatadas com 19 (todas) secretarias municipais envolvidas nessas ações. No que diz respeito às ações com

maior impacto em relação ao número de munícipes 04 ações impactam em toda a população do município pesquisado, são elas; a integração de UBS na proposta de prevenção e promoção da saúde da população considerando as zoonoses de relevância no município, as ações intersetoriais realizadas com as Secretarias, as palestras de prevenção e 
controle do Aedes, e a ação anual de promoção ao uso racional de medicamentos elaborada e implementada.

As ações que apresentam o maior desempenho em relação às metas estabelecidas são respectivamente a ação de cobertura de acompanhamento das condicionalidades de Saúde do Programa Bolsa Família com um percentual de 95\% em relação às metas e a ação dos projetos realizados por equipe de Estratégia de Saúde da Família com 52,94\% de atingimento em relação às metas projetadas.

Diante dos dados, a tabela 3 demonstra os resultados das distâncias positivas e negativas de cada ação assistencial de promoção de saúde pública do município de Araucária em relação a posição ideal.

Tabela 3: Ranking das ações

\begin{tabular}{c|c|c|c|c}
\hline Ação & $\mathbf{D +}$ & $\mathbf{D}-$ & TOPSIS & RANKING \\
\hline 1 & 0,3443 & 0,1441 & 0,295 & 13 \\
\hline 2 & 0,3676 & 0,1141 & 0,237 & 15 \\
\hline 3 & 0,2746 & 0,2068 & 0,430 & 11 \\
\hline 4 & 0,2632 & 0,2123 & 0,447 & 8 \\
\hline 5 & 0,3356 & 0,2644 & 0,441 & 10 \\
\hline $\mathbf{6}$ & $\mathbf{0 , 1 7 6 9}$ & $\mathbf{0 , 3 8 9 0}$ & $\mathbf{0 , 6 8 7}$ & $\mathbf{2}$ \\
\hline 7 & 0,3312 & 0,2655 & 0,445 & 9 \\
\hline $\mathbf{8}$ & $\mathbf{0 , 1 6 8 3}$ & $\mathbf{0 , 3 8 9 7}$ & $\mathbf{0 , 6 9 8}$ & $\mathbf{1}$ \\
\hline 9 & 0,2722 & 0,3308 & 0,549 & 6 \\
\hline 10 & 0,3297 & 0,2024 & 0,380 & 12 \\
\hline 11 & 0,2392 & 0,3040 & 0,560 & 5 \\
\hline $\mathbf{1 2}$ & $\mathbf{0 , 1 9 9 8}$ & $\mathbf{0 , 3 1 6 4}$ & $\mathbf{0 , 6 1 3}$ & $\mathbf{4}$ \\
\hline 13 & 0,2887 & 0,2705 & 0,484 & 7 \\
\hline $\mathbf{1 4}$ & $\mathbf{0 , 1 7 6 9}$ & $\mathbf{0 , 3 8 9 0}$ & $\mathbf{0 , 6 8 7}$ & $\mathbf{3}$ \\
\hline 15 & 0,3627 & 0,1381 & 0,276 & 14 \\
\hline
\end{tabular}

Fonte: Dados da pesquisa, 2019.

De acordo com os indicadores de cada ação foi possível ranqueá-las obtendo uma classificação das mesmas. Assim percebe-se que a ação melhor classificada foi a (ação 8) que trata da ação anual de promoção ao uso racional de medicamentos elaborada e implementada, sendo assim, esta ação teve o melhor desempenho na secretária de saúde do município de Araucária, que entre outros objetivos visa sensibilizar a equipe multiprofissional de saúde com relação ao tema, promovendo e fomentando ações de conscientização e promoção da saúde junto aos usuários, além de envolver um grande número de departamentos a referida ação tem impacto direto potencial em toda a população do município, envolvendo 6 secretarias municipais. Logo a segunda e a terceira melhor ação classificada foi a (ação 6) que trata das ações intersetoriais realizadas com as secretarias, que entre outras atribuições é responsável pela elaboração, impressão e distribuição de materiais técnicos, educativos e de orientação para profissionais e comunidade, tem o papel também de sensibilizar os profissionais das equipes dos órgãos parceiros quanto a importância da vigilância e prevenção de zoonoses para melhoria dos indicadores de saúde local e qualidade de vida da população, promovendo ações que contribuam para elevar a consciência sanitária da sociedade e setor regulado na percepção do risco sanitário, por meio de encontros, seminários, palestras.

Tal ação possui um alto impacto em relação ao número de departamentos envolvidos, totalizando 7 departamentos e com relação direta com 5 secretarias municipais, além do potencial de impacto em toda a população do município. A ação 14 que trata da instituição da gestão municipal do núcleo interno de regulação (NIR) sob responsabilidade 
do departamento de controle, avaliação e auditoria, que tem como principal papel desenvolver a gestão municipal do núcleo interno de regulação, também com um impacto direto em 7 departamentos dentro da secretaria de saúde bem como 5 secretarias do município, a ação 14 tem impacto direto em toda a população do município pesquisado.

Diante da apresentação e discussões dos resultados obtidos a partir da análise, partese para as considerações finais.

\section{CONSIDERAÇÕES FINAIS}

A Gestão da Saúde Pública no Brasil vem sendo feita seguindo o modelo determinado pelos marcos jurídicos e administrativos que norteiam as administrações públicas de municípios, estados e União, mas carece ainda de intenso debate e pesquisa para estabelecer modelos que possam atender com maior eficácia à Gestão da Saúde.

Este artigo teve como objetivo aplicar o método TOPSIS afim de hierarquizar, ranquear indicadores assistenciais de promoção de saúde pública em um município da Região Metropolitana de Curitiba - PR, visando uma melhor análise dos gestores para a tomada de decisão. Tal avaliação é de suma importância pois dinamiza o processo de decisão, fazendo com que os gestores possam perceber quais ações devem ter seus processos priorizados. A partir dos levantamentos das quinze ações (integrantes da diretriz 3 que trata do "Fortalecimento das Ações de Promoção à Saúde") e a identificação dos principais indicadores que medem o desempenho de cada uma delas, foi possível a aplicação do método TOPSIS para a elaboração de um ranking das ações a serem priorizadas pela gestão que se relacionam com indicadores capazes de construir parâmetros de desempenho e importância, dentro do processo de tomada de decisão dos gestores da secretaria saúde.

A pesquisa apontou os resultados das distâncias positivas e negativas de cada ação assistencial de promoção de saúde pública do município de Araucária em relação a posição ideal, trazendo o ranking teve em primeiro lugar ação 8, que trata da de promoção ao uso racional de medicamentos elaborada e implementada com um coeficiente de 0,698 seguida pela ação 6 com coeficiente de 0,687, na sequencia pelas ações 14 e 12 com coeficientes de 0,687 e 0,613 respectivamente. A percepção do ranking é fundamental para direcionar prioridades na gestão da saúde do município uma vez que os diversos recursos (financeiros e não financeiros) disponíveis no município são escassos e necessitam de uma maior eficiência em sua utilização.

Este presente artigo leva em consideração três aspectos, social, empírico e teórico. No aspecto social, este artigo contribui com uma área muito sensível da gestão pública e que impacta significativamente na vida dos cidadãos, pois entende-se que colaborações para dinamizar o processo de gestão nessa área são fundamentais para melhorar o bem esta da população.

Sob o aspecto empírico o presente estudo visa colaborar com outros estudos que têm objetivos correlatos, que em alguma medida buscam contribuir com a construção e desenvolvimento de ferramentas de avaliação de desempenho.

No campo teórico, este estudo colabora com a construção de conhecimento que visa esclarecer ainda mais a necessidade que as organizações possuem em dinamizar os seus processos de tomada de decisão para a eficácia da gestão.

Sugere-se que pesquisas futuras, utilizando outras metodologias de avaliação, bem como outros indicadores, possam avaliar os fatores que venham a impactar na similaridade dos dados, seja na situação ideal positiva, quanto na situação ideal negativa, para que gestores em todas as esferas de atuação em Saúde Pública passem a utilizar ferramentas como esta para controle, avaliação e na tomada de decisão diária. 


\section{REFERÊNCIAS BIBLIOGRÁFICAS}

[1] BULGURCU, Berna Kiran. Application of TOPSIS technique for financial performance evaluation of technology firms in Istanbul stock exchange market. Procedia - Social and Behavioral Sciences, v. 62, n. 1, p. 1033-1040, 2012.

[2] BRASIL. Constituição Federal de 1988. Promulgada em 5 de outubro de 1988. Disponível em: <http://www.planalto.gov.br/ccivil_03/constituicao/constituição.htm> Acesso em: 07 de fev. 2019.

[3] _ Conselho Nacional de Secretários de Saúde. Para entender a gestão do SUS/Conselho Nacional de Secretários de Saúde. - Brasília : CONASS, 2003. 248 p. ISBN 85-89545-02-4 1. SUS (BR). 2. Legislação sanitária. I. Título. NLM WA 525 CDD - 20 ed. 362.1068.

[4] . Decreto no 7.508, de 28 de junho de 2011. Diário Oficial da União, Brasília, n. 123, 29 jun. 2011a. Seção I. p. 1.

[5] _ Lei no 8.080/90, de 19 de setembro de 1990. Diário Oficial da União, Brasília, DF, 20 set. 1990.

[6] — Lei no 8.142 de 28 de dezembro de 1990. Diário Oficial da União, Brasília, DF, 31 dez. 1990.

[7] Lei Complementar no 141, de 13 de janeiro de 2012. Diário Oficial da União, Brasília, n. 11, 16 jan. 2012. Seção I. p.1.

[8] Lei Complementar $n^{\circ}$ 101, de 29 de dezembro de 2000. Diário Oficial da União, Brasília, n. 250, 29 dez. 2000b. Seção I. p. 180.

[9] _. Ministério da Saúde. Sistema de planejamento do SUS: uma construção coletiva. Organização e Funcionamento. 3. ed. Brasília, DF: Ministério da Saúde, 2009a. v. 1.

[10] _ Ministério da Saúde. Secretaria de Atenção à Saúde. Departamento de Regulação, Avaliação e Controle de Sistemas. Critérios e Parâmetros para o Planejamento e Programação de Ações e Serviços de Saúde no âmbito do Sistema Único de Saúde. Brasília, Ministério da Saúde, 2015. Série Parâmetros SUS -Volume 1.

[11] _ Ministério da Saúde. Manual de planejamento no SUS / Ministério da Saúde, Fundação Oswaldo Cruz. - 1. ed., rev. - Brasília : Ministério da Saúde, 2016. 138 p. : il. - (Série Articulação Interfederativa ; v. 4).

[12] _ Ministério da Saúde. Secretaria de Gestão Estratégica e Participativa. A construção do SUS: histórias da Reforma Sanitária e do Processo Participativo / Ministério da Saúde, Secretaria de Gestão Estratégica e Participativa. - Brasília: Ministério da Saúde, 2006. 300 p. - (Série I. História da Saúde no Brasil).

[13] _. Ministério da Saúde. Fundação Oswaldo Cruz ,Manual de planejamento no SUS. 1. ed. Brasília: Editora do Ministério da Saúde, 2016. (Série Articulação Federativa). 
http://bvsms.saude.gov.br/bvs/publicacoes/articulacao_interfederativa _v4_manual_plane jamento_atual.pdf. Acesso em: 15 jun. 2016.

[14] _ _ Ministério da Saúde. Secretaria de Gestão Estratégica Participativa. Caderno de Informações para a Gestão Interfederativa no SUS. Brasília: Editora do Ministério da Saúde, 2012. Disponível em: http://bvsms.saude.gov.br/bvs/publicacoes /caderno_ informacoes_gestao_interfederativa.pdf. Acesso em: 15 jun. 2016.

[15] _. Portaria $n^{\circ}$ 2.135, de 25 de setembro de 2013. Diário Oficial da União, Brasília, n. 187, 26 set. 2013a. Seção I. p. 60.

[16] CAMPOS, Carlos Eduardo Aguilera. O desafio da integralidade segundo as perspectivas da vigilância da saúde e da saúde da família. Ciência \& Saúde Coletiva, Rio de Janeiro, v. 8, n. 2, p. 569- 584, 2003. Disponível em: $<$ http://www.scielo.br/scielo.php?pid=S141381232003000200018\&script=sci_abstract\&tlng=pt> Acesso em: 22 de fev. 2019.

[17] CARVAlHO, Antônio Ivo de; BARBOSA, Pedro Ribeiro. Políticas de saúde: fundamentos e diretrizes do SUS / Antônio Ivo de Carvalho, Pedro Ribeiro Barbosa. Florianópolis: Departamento de Ciências da Administração / UFSC; [Brasília]: CAPES: UAB, 2010. 82p.

[18] CASTRO, Leonardo Mitre Alvim de. Proposição de metodologia para avaliação dos efeitos da urbanização nos corpos de água. 2007. 321 f. Tese (Doutorado em Saneamento, Meio Ambiente e Recursos Hídricos) - Universidade Federal de Minas Gerais.

[19] COLAUTO, Romualdo Douglas; BEUREN, Ilse Maria. Coleta, análise e interpretação dos dados. In: BEUREN, Ilse Maria (Org.). Como elaborar trabalhos monográficos: teoria e prática. São Paulo: Atlas, 2004. p. 117-144.

[20] CONASS. SUS: avanços e desafios./ Conselho Nacional de Secretários de Saúde. Brasília: CONASS, 2006. 164 p.

[21] CONASEMS. Reflexões aos novos gestores municipais de saúde. Conselho Nacional de Secretarias Municipais de Saúde Distrito Federal/Conselho Nacional de Secretarias Municipais de Saúde - Brasília: Conasems, 2009. 200p. Disponível em: $<$ http://bvsms.saude.gov.br/bvs/pu-blicacoes/reflexoes_novos_gestores_municipais .pdf> Acesso em: 17 de fev. 2019.

[22] CUNHA, J.P.P., CUNHA, R.E. Sistema Único de Saúde - SUS: princípios. In: CAMPOS, F.E., OLIVEIRA JÚNIOR, M., TONON, L.M. Cadernos de Saúde. Planejamento e Gestão em Saúde. Belo Horizonte: COOPMED, 1998. Cap.2, p. 11-26.

[23] GOMES, Luiz Flavio A. M.; GOMES, Carlos Francisco S. Tomada de Decisão Gerencial: Enfoque Multicritério. $4^{\circ}$ ed. São Paulo: Editora Atlas, 2012.

[24] HEIN, N.; DEGENHART, L.; VOGT, M.; KROENKE, A.; CAMPESTRINI, Ivan Marcos. Método TOPSIS na avaliação das empresas listadas no IBrX-100: uma avaliação multicritério dos impactos ambientais. In: V CONGRESSO BRASILEIRO DE ENGENHARIA DE PRODUÇÃO, 2015. Anais, Ponta Grossa, PR, 2015.

[25] HELLER, Pedro Gasparini Barbosa. Avaliação dos serviços de saneamento de quatro municípios da bacia hidrográfica do Rio das Velhas - MG: Uma abordagem da dimensão tecnológica. 2007. 139 f. Dissertação (Mestrado em Saneamento, Meio Ambiente e Recursos Hídricos) - Universidade Federal de Minas Gerais. 
[26] JANNUZZI, Paulo de Martino. Indicadores socioeconômicos na gestão pública. Florianópolis: Departamento de Ciências da Administração/UFSC, 2012. p.01-110.

[27] PAIM, Jairnilson Silva; TEIXEIRA, Carmen Fontes. Configuração institucional e gestão do Sistema Único de Saúde: problemas e desafios. Texto elaborado para a Comissão de Políticas, Planejamento e Gestão em Saúde da ABRASCO, como subsídio aos debates da 13a Conferência Nacional de Saúde, outubro de 2007.

[28] MIRANDA, Luiz Miguel. Contribuição a uma modelo de análise multicritério para apoio à decisão da escolha do corredor de transporte para escoamento da produção de granéis agrícolas de Mato Grosso. 2008. 272 f. Tese (Doutorado em Ciências em Engenharia de Transportes) - Universidade Federal do Rio de Janeiro.

[29] RIPSA. Indicadores básicos para a saúde no Brasil: conceitos e aplicações / Rede Interagencial de Informação para a Saúde - Ripsa. - 2. ed. - Brasília: Organização PanAmericana da Saúde, 2008. 349 p.

[30] SILVA, Karolina Cássia Lima; SANTOS, Erlene Roberta Ribeiro; MENDES, Maria do Socorro. O planejamento estratégico no processo de implementação da política de atenção primária em um município da região metropolitana do Recife, Pernambuco, Brasil. Journal of Management and Primary Health Care, Olinda, PE, v. 3, n. 1, p. 1525, 2012.

[31] TEMPORÃO, José Gomes. SUS completa 20 anos com novos desafios. (entrevista) Rev. Saúde pública Santa Catarina, Florianópolis, v. 1, n. 1, jan./jun. 2008 Disponível em: <http://revista.saude.sc.gov.br/index.php/inicio/article/viewFile/4/37> Acesso em: 24 de fev. 2019. 